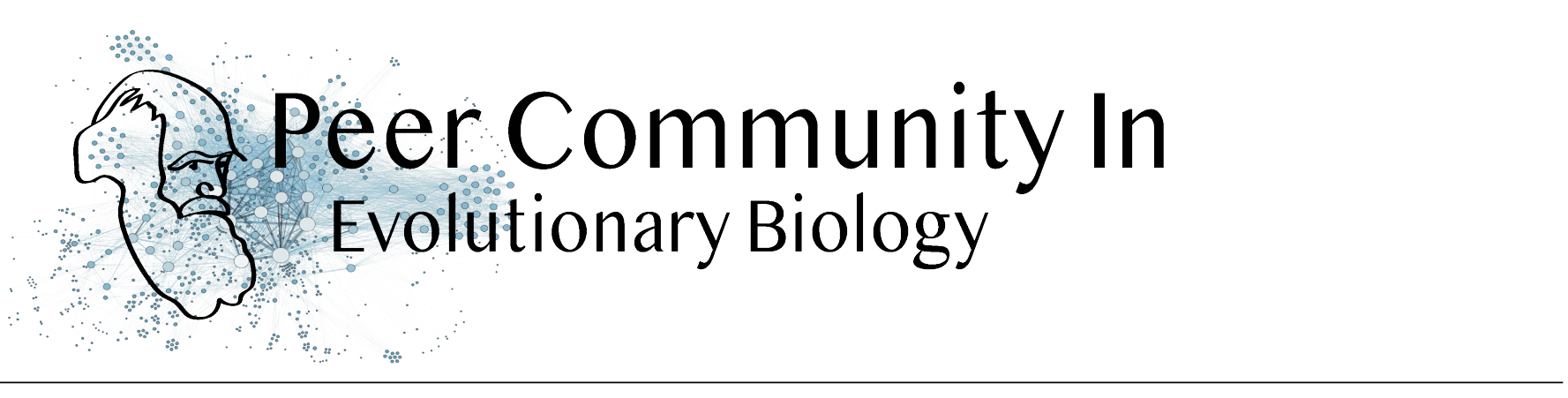

Open Access

RECOMMENDATION

\title{
Inbreeding compensates for reduced sexual selection in purging deleterious mutations
}

Cite as: Baer CF. Inbreeding compensates for reduced sexual selection in purging deleterious mutations. Peer Community In Evolutionary Biology, 100055 (2018). DOI: 10.24072/pci.evolbiol.100055

Published: 8th August 2018

Based on reviews by:

anonymous

anonymous

\section{Correspondence:}

cbaer@ufl.edu

\section{(C) $\ominus$ CC-BY-ND 4.0}

This work is licensed under the Creative Commons Attribution-NoDerivatives 4.0 International License.

\author{
Charles F Baer ${ }^{1}$ \\ ${ }^{1}$ Department of Biology, University of Florida - Gainesville, USA
}

Two evolutionary processes have been shown in theory to enhance the effects of natural selection in purging deleterious mutations from a population (here "natural" selection is defined as "selection other than sexual selection"). First, inbreeding, especially self-fertilization, facilitates the removal of deleterious recessive alleles, the effects of which are largely hidden from selection in heterozygotes when mating is random. Second, sexual selection can facilitate the removal of deleterious alleles of arbitrary dominance, with little or no demographic cost, provided that deleterious effects are greater in males than in females ("genic capture"). Inbreeding (especially selfing) and sexual selection are often negatively correlated in nature. Empirical tests of the role of sexual selection in purging deleterious mutations have been inconsistent, potentially due to the positive relationship between sexual selection and intersexual genetic conflict.

In their preprint, Noël et al. [1] report a cleverly designed, and impressively long-term, experimental evolution study designed to tease apart the relative contributions of selfing and sexual selection in purging deleterious mutations, using the self-compatible hermaphroditic snail Physa acuta. Hermaphroditism relieves at least some of the potential conflict between males and females because each individual expresses traits of each sex. The authors report a 50-generation (ten years!) evolution experiment with four experimental treatments: Control (C), in which snails reproduced by mass mating (allowing sexual selection) and the next generation was sampled randomly from offspring in proportion to maternal family size; Male-selection (M) in which snails reproduced by mass mating but maternal family size was 
held constant, removing the opportunity for fertility selection; Female fertility selection (F) in which snails mated monogamously but fertility selection was imposed, and selfing (S), in which snails reproduced by selfing every other generation, alternating with monogamy + fertility selection. Juvenile survival was taken as the proxy for fitness and was measured for offspring of self-fertilization and of outcross matings. Each line type $(C, M, F, S)$ was replicated twice.

The results are enviably clear-cut: after 50 generations of evolution, outcross fitness dropped precipitously in the $\mathrm{F}$ treatment (monogamy+female fertility selection) and remained at ancestral levels in the other three treatments. Clearly, sexual selection in males is more efficient at purging deleterious alleles than is female fertility selection. Similarly, inbreeding depression was reduced in the $S$ lines relative to the other treatments, indicating that, unsurprisingly, deleterious recessive mutations of large effect are purged under strong inbreeding. Outcross fitness in the $\mathrm{S}$ lines did not decline, in contrast to the $\mathrm{F}$ lines, which indicates that deleterious mutations are on average slightly recessive.

Taken as a whole, this study by Noël et al. [1] provides a compelling empirical demonstration of the efficacy of both sexual selection and strong inbreeding as mechanisms of purging, and implicates sexual conflict as a potentially important factor in studies in which relaxation of sexual selection fails to result in purging.

\section{References}

[1] Noel E, Fruitet E, Lelaurin D, Bonel N, Segard A, Sarda V, Jarne P, and David P. Sexual selection and inbreeding: two efficient ways to limit the accumulation of deleterious mutations. bioRxiv 273367, ver. 3 peer-reviewed by PCI Evol Biol (2018). DoI: 10.1101/ 273367

\section{Appendix}

Reviews by anonymous and anonymous, DOI: 10.24072/pci.evolbiol.100055 